\title{
EVALUATION OF PERMANENTS
}

\author{
by HENRYK MINC ${ }^{\dagger}$ \\ (Received 7th September 1976, revised 4th July 1977)
}

\section{Introduction}

The permanent of an $m \times n$ matrix $A=\left(a_{i j}\right), m \leqslant n$, is defined by

$$
\operatorname{Per}(A)=\sum_{\sigma} \prod_{i=1}^{m} a_{i \sigma(i)}
$$

where the summation is over all one-to-one functions $\sigma$ from $\{1, \ldots, m\}$ to $\{1, \ldots, n\}$. In other words, the permanent of $A$ is the sum of all the diagonal products of $A$, that is, all the products of $m$ entries of $A$ no two of which lie in the same row or in the same column. Thus the permanent of $A$ may be evaluated by first multiplying all the row sums of $A$ and then subtracting from the product all terms that contain as factors two or more entries from the same column of $A$. This is the idea behind the formulas of Binet and of Ryser.

Permanents were introduced in 1812 by Binet (1) and Cauchy (2). Nearly all papers on permanents written in the succeeding century and a half dealt only with permanents of square matrices; in fact, the permanent function was regarded as a counterpart of the determinant function. The interest in permanents of rectangular matrices has revived in recent years mainly due to the work of Ryser who obtained a formula for the evaluation of permanents based on the principle of inclusion and exclusion (4). The same principle was used by Binet (1) to develop formulas for permanents of $m \times n$ matrices for $m=2,3$ and 4 . In this paper I extend Binet's formulas to all values of $m, m \leqslant n$.

\section{NOTATION}

Let $Q_{s, k}$ denote the set of increasing sequences of integers $\omega=\left(\omega_{1}, \ldots, \omega_{s}\right)$, $1 \leqslant \omega_{1}<\cdots<\omega_{s} \leqslant k$, and let $G_{s, k}$ denote the set of nondecreasing sequences of integers $\omega=\left(\omega_{1}, \ldots, \omega_{s}\right), 1 \leqslant \omega_{1} \leqslant \cdots \leqslant \omega_{s} \leqslant k$. For an $m \times n$ matrix $A=\left(a_{i j}\right), m \leqslant n$, and a sequence $\left(i_{1}, \ldots, i_{s}\right) \in Q_{s, m}$, let $r_{i_{1} * i_{2} * \cdots * i_{s}}$ denote the sum of the entries in the Hadamard product of rows $i_{1}, \ldots, i_{s}$ of $A$, i.e.,

$$
r_{i_{1} * i_{2} * \cdots * i_{s}}=\sum_{j=1}^{n} a_{i_{1} j} a_{i_{2} j} \cdots a_{i_{j}} \text {. }
$$

In particular, $r_{i}$ or $r_{i}(A)$ denotes the $i$-th row sum of $A$,

$$
r_{i}=\sum_{j=1}^{n} a_{i j}
$$

${ }^{+}$This research was supported by the Air Force Office of Scientific Research under grant \# 72-2164. 
Let $D(m)$ be the set of increasing sequences of positive integers $\left(t_{1}, \ldots, t_{k}\right)$ such that $t_{1}+\cdots+t_{k}=m$. For $\left(t_{i}, \ldots, t_{k}\right) \in D(m)$ the $R$-function $R\left(t_{1}, \ldots, t_{k}\right)$ is the symmetrised sum of all distinct products of the $r_{i_{1} * \cdots * i_{t_{j}}}$ so that in each product the sequences $\left(i_{1}, \ldots, i_{i_{j}}\right) \in Q_{i_{j}, m}, j=1, \ldots, k$, partition the set $\{1, \ldots, m\}$. For example,

$R(1,1)=r_{1} r_{2}, \quad R(2)=r_{1 * 2}$;

$R(1,1,1)=r_{1} r_{2} r_{3}, \quad R(1,2)=r_{1} r_{2 * 3}+r_{2} r_{1 * 3}+r_{3} r_{1 * 2}, \quad R_{3}=r_{1 * 2 * 3} ;$

$R(1,1,1,1)=r_{1} r_{2} r_{3} r_{4}$,

$R(1,1,2)=r_{1} r_{2} r_{3 * 4}+r_{1} r_{3} r_{2 * 4}+r_{1} r_{4} r_{2 * 3}+r_{2} r_{3} r_{1 * 4}+r_{2} r_{4} r_{1 * 3}+r_{3} r_{4} r_{1 * 2}$,

$R(1,3)=r_{1} r_{2 * 3 * 4}+r_{2} r_{1 * 3 * 4}+r_{3} r_{1 * 2 * 4}+r_{4} r_{1 * 2 * 3}$,

$R(2,2)=r_{1 * 2} r_{3 * 4}+r_{1 * 3} r_{2 * 4}+r_{1 * 4} r_{2 * 3}, \quad R(4)=r_{1 * 2 * 3 * 4}$, etc.

The number of products $r_{i_{1} * \ldots * i_{s}}, s=t_{1}, \ldots, t_{k}$, in $R\left(t_{1}, \ldots, t_{k}\right)$ is $m ! / \Pi_{i=1}^{k} t_{i} !\left(m_{i} !\right)^{1 / m_{i}}$ where $m_{i}$ denotes the multiplicity of $t_{i}$ in the sequence $\left(t_{1}, \ldots, t_{k}\right)$.

If $\alpha \in Q_{k, m}$ and $A$ is an $m \times n$ matrix, then $A(\alpha \mid-)$ denotes the $(m-k) \times n$ submatrix of $A$ obtained from $A$ by deleting rows indexed with $\alpha$. Similarly if $\beta \in Q_{h, n}$, then $A(-\mid \beta)$ denotes the $m \times(n-h)$ submatrix of $A$ obtained from $A$ by deleting columns indexed with $\beta$.

\section{Ryser's formula}

Let $A$ be an $m \times n$ matrix, $m \leqslant n$, and let $\Lambda_{k}$ denote the totality of $m \times k$ submatrices of $A$, that is,

$$
\Lambda_{k}=\left\{A(-\mid \beta) \mid \beta \in Q_{k, n}\right\}
$$

Ryser's formula (4) states:

$$
\begin{aligned}
\operatorname{Per}(A)= & \sum_{X \in \Lambda_{m}} \prod_{i=1}^{m} r_{i}(X)-\left(\begin{array}{c}
n-m+1 \\
1
\end{array}\right) \sum_{X \in \Lambda_{m-1}} \prod_{i=1}^{m} r_{i}(X) \\
& +\cdots+(-1)^{r}\left(\begin{array}{c}
n-m+r \\
r
\end{array}\right) \sum_{X \in \Lambda_{m-r}} \prod_{i=1}^{m} r_{i}(X)+\cdots+(-1)^{m-1}\left(\begin{array}{c}
n-1 \\
m-1
\end{array}\right) \sum_{X \in \Lambda_{1}} \prod_{i=1}^{m} r_{i}(X) .
\end{aligned}
$$

Ryser's formula (1) can be modified so that the first summand is replaced by the product of the row sums of $A$ :

$$
\operatorname{Per}(A)=\prod_{i=1}^{m} r_{i}(A)+\sum_{i=1}^{m-1} c_{t} \sum_{x \in \Lambda_{m-t}} \prod_{i=1}^{m} r_{i}(X),
$$

where $c_{1}=-1$ and $c_{t}=-1-\sum_{r=1}^{t-1}\left(\begin{array}{c}n-m+t \\ t-r\end{array}\right) c_{n} t=2, \ldots, m-1$.

We prove the formula (2). The first summand on the right-hand side, $\prod_{i=1}^{m} r_{i}(A)$, contains all the diagonal products of $\operatorname{Per}(A)$ and, in addition, products involving two or more entries from the same column. The other summands, $\prod_{i=1}^{m} r_{i}(X), X \in \Lambda_{m-l}$, $t=1, \ldots, m-1$, contain only products of the second type, viz., $a_{i \omega_{1}} a_{2 \omega_{2}} \ldots a_{m \omega_{m}}$ with $\omega=\left(\omega_{1}, \ldots, \omega_{m}\right) \in G_{m, n}-Q_{m, n}$. We show that for each such product the sum of 
coefficients on the right-hand side of (2) is 0 . Let $\omega=\left(\omega_{1}, \ldots, \omega_{m}\right) \in G_{m, n}$ be a nondecreasing sequence with exactly $k$ distinct values for the $\omega_{i}, 1 \leqslant k \leqslant m-1$. The product $a_{1 \omega_{1}} a_{2 \omega_{2}} \cdots a_{m \omega_{m}}$ appears once in $\Pi_{i=1}^{m} r_{i}(A)$ and once in each summand $\Pi_{i=1}^{m} r_{i}(X)$ for which the matrix $X$ contains the $k$ columns of $A$ numbered $\omega_{1}, \ldots, \omega_{m}$; there are $\left(\begin{array}{c}n-k \\ m-t-k\end{array}\right)$ such matrices $X$ in $\Lambda_{m-t}, t=1, \ldots, m-k$. Hence the sum of the coefficients of $a_{1 \omega_{1}} a_{2 \omega_{2}} \cdots a_{m \omega_{m}}$ on the right-hand side of (2) is

$$
1+\sum_{t=1}^{m-k}\left(\begin{array}{c}
n-k \\
m-t-k
\end{array}\right) c_{t}
$$

But

$$
\begin{aligned}
1+\sum_{t=1}^{m-k}\left(\begin{array}{c}
n-k \\
m-t-k
\end{array}\right) c_{t} & =1+c_{m-k}+\sum_{r=1}^{m-k-1}\left(\begin{array}{c}
n-m+(m-k) \\
(m-k)-r
\end{array}\right) c_{r} \\
& =1+c_{m-k}+\left(-1-c_{m-k}\right) \\
& =0,
\end{aligned}
$$

by the definition of the $c_{t}$ in (2).

If $m=n$, then the formulas (1) and (2) both become

$$
\operatorname{Per}(A)=\sum_{i=0}^{n-1}(-1)^{t} \sum_{X \in \Lambda_{n-1}} \prod_{i=1}^{n} r_{i}(X) .
$$

Example. If

$$
A=\left[\begin{array}{rrrrr}
1 & 2 & 3 & 4 & 5 \\
1 & 0 & -1 & 0 & 1 \\
5 & 4 & 3 & 2 & 1
\end{array}\right]
$$

then

$$
\sum_{X \in \Lambda_{3}} r_{1}(X) r_{2}(X) r_{3}(X)=474, \quad \sum_{X \in \Lambda_{2}} r_{1}(X) r_{2}(X) r_{3}(X)=126
$$

and

$$
\begin{aligned}
\sum_{X \in \Lambda_{1}} r_{1}(X) r_{2}(X) r_{3}(X)=1 . \quad \text { Thus, by (1) } \\
\operatorname{Per}(A)=474-\left(\begin{array}{c}
5-3+1 \\
1
\end{array}\right) \cdot 126+\left(\begin{array}{c}
5-3+2 \\
2
\end{array}\right) \cdot 1 \\
=102
\end{aligned}
$$

and by (2),

$$
\begin{aligned}
\operatorname{Per}(A) & =15 \cdot 1 \cdot 15-126+\left(-1+\left(\begin{array}{c}
5-3+2 \\
1
\end{array}\right)\right) \cdot 1 \\
& =102 .
\end{aligned}
$$




\section{Binet's formulas}

Binet's formulas (1) for permanents are also based on the principle of inclusion and exclusion. They start with the product of the row sums of the matrix. The superfluous terms are then removed by a method different from that used by Ryser.

Let $A=\left(a_{i j}\right)$ be an $m \times n$ matrix, $m \leqslant n$. If $m=2$, then it is easy to see that

$$
\begin{aligned}
\operatorname{Per}(A) & =\sum_{s \neq t} a_{1 s} a_{2 t} \\
& =r_{1} r_{2}-r_{1} * 2 \\
& =R(1,1)-R(2) .
\end{aligned}
$$

This is Binet's formula for the permanents of $2 \times n$ matrices.

If $A=\left(a_{i j}\right)$ is a $3 \times n$ matrix, $3 \leqslant n$, we start again with the product $r_{1} r_{2} r_{3}$ which contains all the terms of $\operatorname{Per}(A)$ and in addition "unwanted" terms such as $a_{11} a_{21} a_{3 j}$, $a_{12} a_{22} a_{3 j}, \ldots, a_{11} a_{2 j} a_{31}, \ldots, a_{1 j} a_{21} a_{31}, \ldots, j=1, \ldots, n$; that is, the terms of $r_{1 * 2} r_{3}, r_{1 * 3} r_{2}$ and $r_{2 * 3} r_{1}$. Hence

$$
r_{1} r_{2} r_{3}-\left(r_{1 * 2} r_{3}+r_{1 * 3} r_{2}+r_{2 * 3} r_{1}\right)
$$

does not contain any products involving exactly two entries from the same column. However, the terms $a_{1 j} a_{2 j} a_{3 j}, j=1, \ldots, n$, appear once in each of $r_{1 * 2} r_{3}, r_{1 * 3} r_{2}, r_{2 * 3} r_{1}$ and therefore each of these has been subtracted three times. We off set this by adding $2 r_{1 * 2 * 3}$ and thus we obtain

$$
\begin{aligned}
\operatorname{Per}(A) & =r_{1} r_{2} r_{3}-\left(r_{1 * 2} r_{3}+r_{1 * 3} r_{2}+r_{2 * 3} r_{1}\right)+2 r_{1 * 2 * 3} \\
& =R(1,1,1)-R(1,2)+2 R(3) .
\end{aligned}
$$

Example. If $A$ is the matrix in the example in Section 3, then by formula (5),

$$
\begin{aligned}
\operatorname{Per}(A) & =15 \times 1 \times 15-(3 \times 15+35 \times 1+3 \times 15)+2 \times 1 \\
& =102 .
\end{aligned}
$$

Binet also gave a formula for the permanents of $4 \times n$ matrices, $4 \leqslant n$ :

$$
\operatorname{Per}(A)=R(1,1,1,1)-R(1,1,2)+2 R(1,3)+R(2,2)-6 R(4) .
$$

It is easy to reason out the above formula by the method of inclusion and exclusion. In fact, it is not difficult to obtain by similar direct ad hoc methods formulas for the permanents of $m \times n$ matrices, $m \leqslant n$, for the next few values for $m$. However, the procedure becomes progressively more tedious and prohibitively complicated for larger values of $\mathrm{m}$. Binet himself gave only formulas (4), (5) and (6) without suggesting how they can be extended to larger values of $m$ and without indicating how the coefficients can be derived. In the next section I give new recursive formulas for the permanents of general $m \times n$ matrices, $m \leqslant n$. The method is based to a certain extent on Joachimstal's (3) proof of Binet's formulas. 


\section{New results}

Let $\omega=\left(i_{1}, \ldots, i_{k}\right) \in Q_{k, m-1}$. We shall use the abbreviated notation $r_{\omega * m}$ for $r_{i_{1}} * \cdots * i_{k} * m \cdot$

Theorem 1. Let $A=\left(a_{i j}\right)$ be an $m \times n$ matrix, $2 \leqslant m \leqslant n$. Then

$$
\operatorname{Per}(A)=\operatorname{Per}(A(m \mid-)) r_{m}+\sum_{k=1}^{m-1}(-1)^{k} k ! \sum_{\omega \in \mathbb{Q}_{k, m-1}} \operatorname{Per}(A(\omega, m \mid-)) r_{\omega * m},
$$

where $\operatorname{Per}(A(\omega, m \mid-))$ is interpreted as 1 for $\omega=(1, \ldots, m-1)$.

Proof. Each of terms of the expansion of $\operatorname{Per}(A)$ appears in the first summand on the right-hand side of $(7), \operatorname{Per}(A(m \mid-)) r_{m}$, with coefficient 1 , and it does not appear at all in the other summands. The other terms in various summands on the right-hand side of (7) are of the form

$$
a_{i, j} a_{i_{2} j} \cdots a_{i_{j} j} a_{m j} \prod_{t=s+1}^{m-1} a_{i, j}
$$

where $1 \leqslant s \leqslant m-1,\left(i_{1}, \ldots, i_{m-1}\right)$ is a permutation of $(1, \ldots, m-1)$ and $j, j_{s+1}, \ldots, j_{m-1}$ are distinct integers in $\{1, \ldots, n\}$. It remains to show that the sum of the coefficients of each term (8) in the summands

$$
(-1)^{k} k ! r_{\omega * m} \operatorname{Per}(A(\omega, m \mid-)) \text {, }
$$

$\omega \in Q_{k, m-1}, k=0,1, \ldots, m-1$, is zero (for $k=0$ the expression (9) is interpreted as $r_{m} \operatorname{Per}(A(m \mid-))$. Each term (8) appears exactly once in $r_{\omega * m}(\operatorname{Per}(A(\omega, m \mid-))$ where $\omega=\left(i_{1}, \ldots, i_{s}\right) \in Q_{s, m-1}$, and exactly once in each of the $s$ summands $r_{\omega * m} \operatorname{Per}(A(\omega, m \mid-))$ if $\omega \in Q_{s-1, m-1}$ is a subsequence of $\left(i_{1}, \ldots, i_{s}\right)$. Thus the term (8) appears on the right-hand side of (7) with coefficient

$$
(-1)^{s} s !+s\left((-1)^{s-1}(s-1) !\right)=0 .
$$

Formula (7) can be used to derive an explicit formula for the permanent of an $m \times n$ matrix $A$ in terms of the functions $R(\omega)$. Of course, the coefficients in the formula still depend on partitions of $m$.

Theorem 2. Let $A$ be an $m \times n$ matrix, $2 \leqslant m \leqslant n$. Then

$$
\operatorname{Per}(A)=\sum_{\omega \in D(m)} c(\omega) R(\omega)
$$

where

$$
\omega=\left(\omega_{1}, \ldots, \omega_{k}\right) \in D(m) \text { and } c(\omega)=(-1)^{m+k} \prod_{i=1}^{k}\left(\omega_{i}-1\right) !
$$

Proof. Formula (10) states that $\operatorname{Per}(A)$ is a linear combination of the $R$-functions. This is an immediate consequence of Theorem 1 , by virtue of the symmetry of the permanent function with respect to rows of the matrix. It remains to prove formula 
(11) which gives the coefficients $c(\omega)$. Let $\omega=\left(\omega_{1}, \ldots, \omega_{k}\right) \in D(m)$. If $k=1$, then $R(\omega)=r_{1}+2 * \cdots, m$ and, by (7), $c(\omega)=(-1)^{m-1}(m-1)$ !. We now use induction on $k$. Let $\alpha \in Q_{\omega_{1}-1, m-1}$ and consider the terms of $r_{\alpha * m} \operatorname{Per}\left(A(\alpha, m \mid-)\right.$ ) (or of $r_{m} \operatorname{Per}(A(m \mid-)$ ) in case $\omega_{1}=1$ ) that belong to $R(\omega)$. They are the terms of $r_{a, m} R\left(\omega_{2}, \ldots, \omega_{k}\right)$ (or of $\left.r_{m} R\left(\omega_{2}, \ldots, \omega_{k}\right)\right)$, and they appear with the coefficient $(-1)^{\omega_{1}-1}\left(\omega_{1}-1\right) ! c\left(\omega_{2}, \ldots, \omega_{k}\right)$ which, by symmetry, is the coefficient of $R(\omega)$ in (10). Hence, using the induction hypothesis,

$$
\begin{aligned}
c(\omega) & =(-1)^{\omega_{1}-1}\left(\omega_{1}-1\right) ! c\left(\omega_{2}, \ldots, \omega_{k}\right) \\
& =(-1)^{\omega_{1}-1}\left(\omega_{1}-1\right) !(-1)^{m-\omega_{1}+k-1} \prod_{i=2}^{k}\left(\omega_{i}-1\right) ! \\
& =(-1)^{m+k} \prod_{i=1}^{k}\left(\omega_{i}-1\right) ! .
\end{aligned}
$$

The author is indebted to Mr. Michael Tarsi for suggesting the explicit form of formula (11).

\section{REFERENCES}

(1) J. BiNET, Mémoire sur un système de formules analytiques, et leur application à des considérations géométriques, J. de l'Éc. Polyt. 9 (1812), 280-302.

(2) A. L. CAUCHY, Mémoire sur les fonctions qui ne peuvent obtenir que deux valeurs égales et de signes contraires par suite des transpositions opérées entre les variables qu'elles renferment, J. de l'Éc. Polyt. 10 (1812), Cah. 17, 29-112; Oeuvres (2)i.

(3) F. JOACHIMSTAL, De aequationibus quarti et sexti gradus quae in theoria linearum et superficierum secundi gradus occurunt, Crelle's J., 53 (1856), 149-172.

(4) H. J. RYSER, Combinatorial Mathematics (Carus Math. Monograph No. 14, 1963).

UNIVERSITY OF CALIFORNIA

SANTA BARBARA 